\title{
Predicting economic market crises using measures of collective panic
}

\author{
Dion Harmon ${ }^{1}$, Marcus A. M. de Aguiar ${ }^{1,2}$, David D. \\ Chinellato $^{1,2}$, Dan Braha ${ }^{1,3}$, Irving R. Epstein ${ }^{1,4}$, Yaneer Bar-Yam ${ }^{1}$ \\ ${ }^{1}$ New England Complex Systems Institute, \\ 238 Main St., Suite 319, Cambridge, MA 02142, USA \\ ${ }^{2}$ Universidade Estadual de Campinas, \\ Unicamp 13083-970, Campinas, SP, Brasil \\ 3 University of Massachusetts Dartmouth, Dartmouth, MA 02747 \\ 4 Brandeis University, MS 015 Waltham MA, 02454
}

(Dated: August 26, 2010; revised February 13, 2011)

\begin{abstract}
Predicting panic is of critical importance in many areas of human and animal behavior, notably in the context of economics. The recent financial crisis is a case in point. Panic may be due to a specific external threat, or self-generated nervousness. Here we show that the recent economic crisis and earlier large single-day panics were preceded by extended periods of high levels of market mimicry - direct evidence of uncertainty and nervousness, and of the comparatively weak influence of external news. High levels of mimicry can be a quite general indicator of the potential for selforganized crises.
\end{abstract}


In sociology [1 4], panic has been defined as a collective flight from a real or imagined threat. In economics, bank runs occur at least in part because of the risk to the individual from the bank run itself - and may be triggered by predisposing conditions, external (perhaps catastrophic) events, or even randomly [5, 6]. Although empirical studies of panic are difficult [7 9], efforts to distinguish endogenous (self-generated) and exogenous market panics from oscillations of market indices have met with some success [10-14], though the conclusions have been debated [15 18]. Market behavior is often considered to reflect external economic news, though empirical evidence has been presented to challenge this connection [19]. Efforts to characterize events range from the Hindenburg Omen [20] to microdynamic models [21] and to the demonstration that market behaviors are invariant across many scales [22]. Other work has looked at relationships of market behavior with internet search [23]. Panic can be considered a 'critical transition' for which early warnings are being sought [24]. The "collective flight" aspect of such a transition should be revealed in measures of mimicry that is considered central to panic. Here we use co-movement data to evaluate whether the recent market crisis and earlier one-day crashes are internally generated or externally triggered. Based upon a hypothesis about mimicry, we construct a model that includes both mimicry and external factors and test it empirically against the daily extent of co-movement. Our objective is to determine the relative importance of internal and external causes, and, where internal causes are important, to find a signature of self-induced panic, which can be used to predict panic.

The literature generally uses volatility and the correlation between stock prices to characterize risk. 25 31] These measures are sensitive to the magnitude of price movement and therefore increase dramatically when there is a market crash. Studies find that, on average, volatility increases following price declines, but do not show higher volatility is followed by price declines. 32 36] We are interested in the extent to which stocks move together. The extent of such co-movement may be large even when price movements are small. Indeed, even when price changes are small, we expect that co-movement itself is the collective behavior that is characteristic of panic, or panicky behavior that precedes a panic. Thus, rather than measuring volatility or correlation, we measure the fraction of stocks that move in the same direction. We find that this increases well before the market crash, and there is significant advance warning to provide a clear indicator of an impending crash. The existence of the indicator shows that market crashes are preceded by nervousness that gives rise to following 
behavior — increased collective behavior prior to a panic.

We consider the "co-movement" of stocks over time by plotting the number of days in a year that a particular fraction of the market moves up (the complement moving down). Intuitively, if substantially more or less than $50 \%$ of the market moves in the same direction, this represents co-movement. As shown in Fig. 1, the results indicate that in 2000, the curve is peaked near $1 / 2$, so that approximately $50 \%$ of stocks are moving up or down on any given day. Over the decade of the 2000s, however, the curve became progressively flatter - in 2008 the likelihood of any fraction is almost the same for any value. The probability that a large fraction of the market moves in the same direction, either up or down, on any given day, increased dramatically. Such high levels of co-movement may manifest the collective behavior we are searching for.

To quantitatively describe co-movement, we start from a behavioral economics model of a single stock that describes trend-following "bandwagons." It has been shown that investors can benefit from trend-following [37-40]. Moreover, there is no need for the change to be based upon fundamental value for it to provide benefit to the investors [37, 38]. When individuals observe that a stock increases (decreases) in value, and choose to buy (sell) in anticipation of future increases (decreases), this self-consistently generates the desired direction of change. Such a "bandwagon" effect can undermine the assumptions of market equilibrium. We hypothesize that this trend-following mimicry across multiple stocks can cause a marketwide panic, and we build a model to capture its signature. We assume that investors in a stock observe three things, the direction of their stock, external indicators of the economy, and the direction of other stocks. The last of these is the potential origin of self-induced market-wide panic.

To model the co-movement fraction, we represent only whether a stock value rises or falls. This enables us to directly characterize the degree to which stocks move together and not how far they move at any particular time. Stocks are represented by nodes of a network and influences between stocks by links between nodes, an appropriate representation for market analysis [27 29, 41]. We consider both fully and partly connected networks. Every day, each of the $N$ nodes is labeled by a sign $(+/-)$ indicating the daily return of the stock. Market dynamics is simulated by randomly selecting nodes, which maintain their current sign or randomly copy the sign of one of their connected neighbors. To represent external influences, we add nodes that influence others, but are not themselves influenced, i.e. "fixed" 

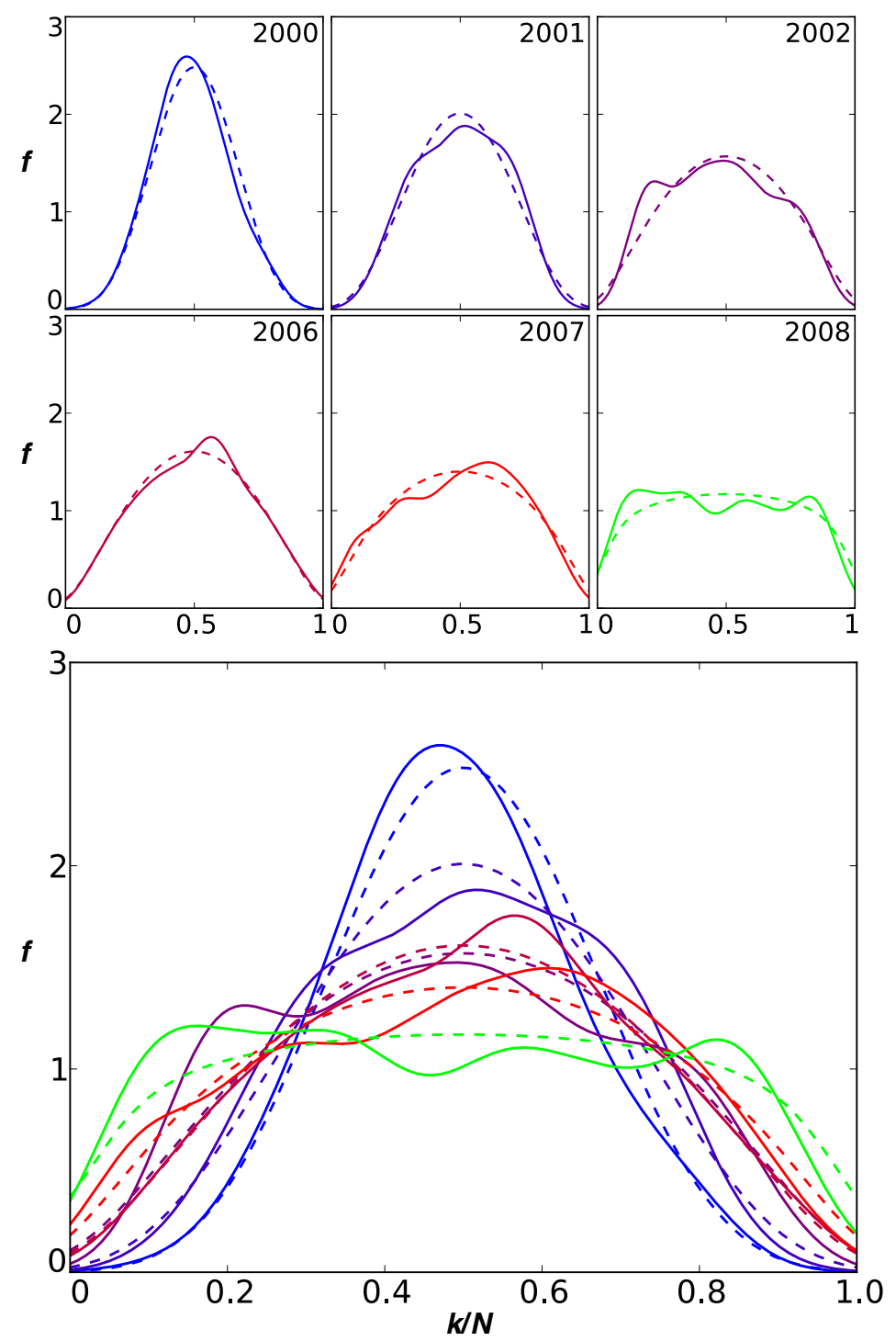

FIG. 1: The co-movement of stocks. Plotted is the fraction of trading days during the year $(f$, vertical axis) in which a certain fraction of stocks $(k / N$, horizontal axis) moved up. Empirical data are shown (solid lines) along with one-parameter theoretical fits (dashed lines) for the years indicated. Three years are omitted that do not differ much from the year immediately preceding and after them. Bottom panel combines all of the years shown. Stocks included are from the Russell 3000 that trade on the NYSE or Nasdaq. Curves are kernel density estimates with Gaussian kernels $(\sigma=0.06)$. Fits pass the $\chi^{2}$ goodness-of-fit test (the deviation of the data from the theoretical distribution is not statistically significant at the $25 \%$ level).

nodes. The number of fixed nodes influencing in a positive direction is $U$ and the number influencing in a negative direction is $D$. The effective strength of the positive and negative external influences is given by the number of these nodes. External influences of opposite types do not cancel; instead larger $U$ and $D$ reflect increasing probability that external 
influences determine the returns of a stock independent of the changes in other stocks. This is the conventional view that news is responsible for the market behavior. Good news would be represented by $U$ greater than $D$, bad news by $D$ greater than $U$.

We have previously proposed this model as a widely applicable theory of collective behavior of complex systems. [42] Successful matching to data will be a confirmation of this theory. It has also been previously identified as a model of conformity and non-conformity in social systems, [43] and it has been studied in application to evolutionary dynamics. 44]

This model can be solved exactly for a fully connected network (see Appendix). We obtain the probability of a co-movement fraction:

$$
f(k / N)=\frac{\left(\begin{array}{c}
U+k-1 \\
k
\end{array}\right)\left(\begin{array}{c}
N+D-k-1 \\
N-k
\end{array}\right)}{\left(\begin{array}{c}
N+D+U-1 \\
N
\end{array}\right)}
$$

where $\mathrm{N}$ is the number of stocks, $k$ is the number of stocks with positive returns and $\left(\begin{array}{l}n \\ k\end{array}\right)$ are binomial coefficients. The behavior is controlled by the strength of external stimuli, $U$ and D, compared to the strength of interactions within the network, and the relative bias of the external influence toward positive, $\mathrm{U}$, or negative, D, effects. When interactions are weak compared to external forces $(D, U>>1)$, the distribution is essentially normal. When internal interactions are strong (small $D, U$ ), the distribution is neither normal nor longtailed. Instead it becomes flatter, becoming exactly flat at the critical value $(D=U=1)$, where the external influences only have the strength of a single node. Analytic continuation allows $U$ and $D$ to be extended to non-integer values. There are three parameters of the distribution, $D, U, N$, but the third is fixed to the number of stocks. We can compare this to the binomial or normal distributions, which are specified by two parameters, the average and standard deviation. The distribution we obtain has a wider range of behaviors, and the normal distribution arises as a limiting case.

If we consider a more complete model of influences, in which investors of one stock only consider specific other stocks as guides, we have a partly connected network. We have studied the dynamics of such networks analytically and through simulations, and the primary modification from fully-connected networks is to amplify the effect of the external influences (see Appendix). As the links within the network are fewer, the network can be approximated by a more weakly coupled, fully connected network, with a weakening factor given by the 
average number of links compared to the number of possible links. Similarly, if only a subset of the external influences are considered relevant for the return of a specific stock, the relative strength of the external influences can be replaced by weaker, uniform external influences. Otherwise, for many cases, the shape of the distribution is not significantly affected. The model thus measures the relative strengths of the internal and external influences rather than the absolute strength of either. The model's robustness indicates a universality across a wide range of network topologies, suggesting applicability to real world systems.

Compared with recent empirical market data in Fig. 1, the model fits remarkably well. A Gaussian model fits the early years, less well in the final years, and does not fit 2008. The good agreement of our model is obtained with equal up and down influences, $U=D$, which is the only adjustable parameter. This implies that whether the market value is trending up or down, or has large one day drops, over a period of a year co-movements occur symmetrically in both up and down directions. Parameter values are given in Table I.

\begin{tabular}{|c|c|}
\hline Year & $U=D$ \\
\hline 2000 & 5.79 \\
\hline 2001 & 3.66 \\
\hline 2002 & 2.21 \\
\hline 2006 & 2.32 \\
\hline 2007 & 1.77 \\
\hline 2008 & 1.24 \\
\hline
\end{tabular}

TABLE I: Model parameter values used to generate the distributions in Figure 1. Empirically, we find that stock return distributions are symmetric, reducing our model to only one free parameter, $D=U$. Similar results are obtained using direct fits and by using the standard deviation of the distribution (see Appendix).

The economic crisis period's flat distribution corresponds to $D=U=1$. This is the critical value of the model where external influences are very weak compared to the influences among stocks as a whole. By contrast, predominantly negative effects, $D>U$, would manifest as a distribution whose mean is shifted to the left. Thus, rather than negative news, uncertainty and collective mimicry led to a self-organized crash.

The flattening of the stock market distribution may serve as a measure of market vulnerability to panic, and the projection of a flat distribution observed in the economic crisis can be used as an early warning signal. Figure 2 shows the empirical results of the single parameter from 2000-2010. We note that the average used for the value at any point of time 


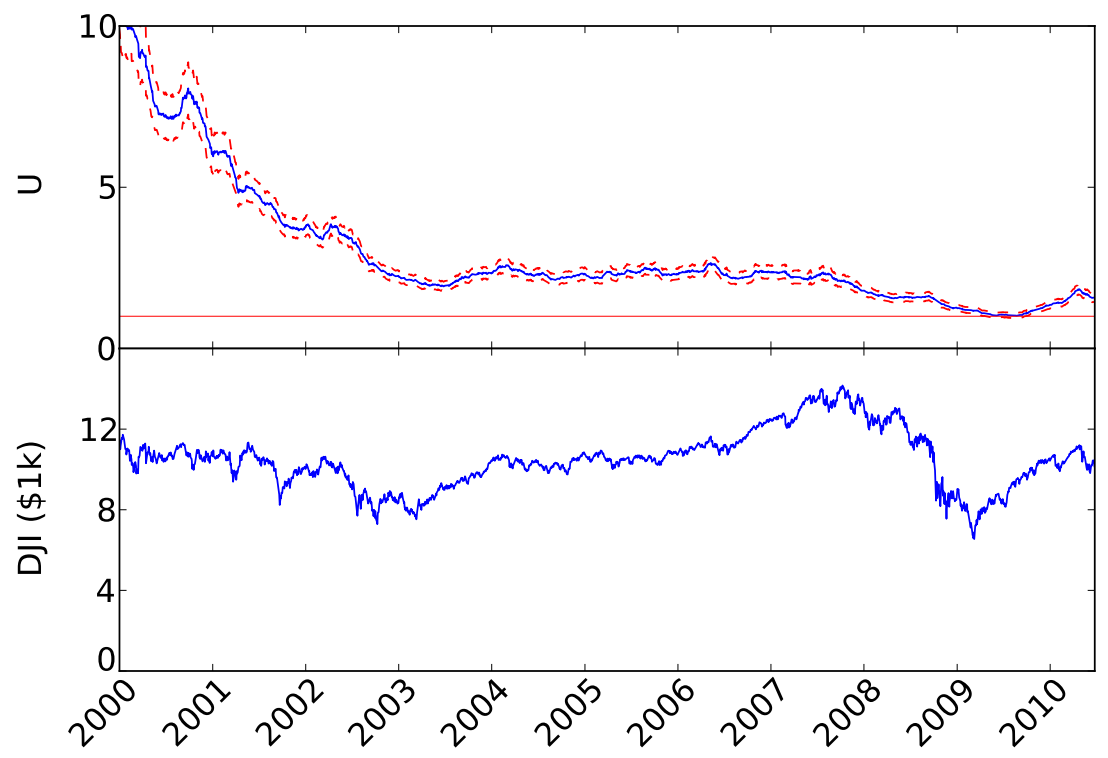

FIG. 2: Model parameter (top panel) and the Dow Jones Industrial Average (bottom panel) for the period 2000-2010. Estimates of the model parameter are shown at the end of the year-long period for which $U$ was estimated. Sampling error estimates are drawn at \pm 1 standard deviations. Positive-return distributions are computed from the daily returns of stocks of the Russell 3000.

is from the period of 12 months prior to that time in order to evaluate the predictive ability. A significant drop occurred in the 2000-2002 period, followed by a plateau that declined gradually beginning in mid-2007 until it hit the critical value at $U=1$. This suggests the market was vulnerable well before the financial crisis, and the gradual decrease before the crisis suggests that the crisis could have been anticipated.

In order to evaluate more broadly the predictive ability of the model, we consider the period from 1985-2010 (Figure 3). While there was no other financial crisis of comparable magnitude to the current one, drops in the model parameter $U$ anticipate large drops of the Dow Jones Industrial Average (DJI). The bottom panel of Figure 3 shows the (annual) change in the model parameter as a fraction of the standard deviation one year earlier, averaged over the preceding year. Of the twenty largest percentage drops of the DJI, eight are in the displayed time period [45, proximate to Black Monday [46, the Asian market crisis [47, September 11, 2001, and the recent financial crisis. A simple signature pattern precedes the drops by less than a year: after a period of positive change, a large drop occurs in the parameter $U$, greater than twice the standard deviation from one year earlier, when averaged over the preceding year. This pattern identifies four year-long windows in which occur the eight largest percentage drops of the DJI within the last 26 years $(p<0.00007$ for 

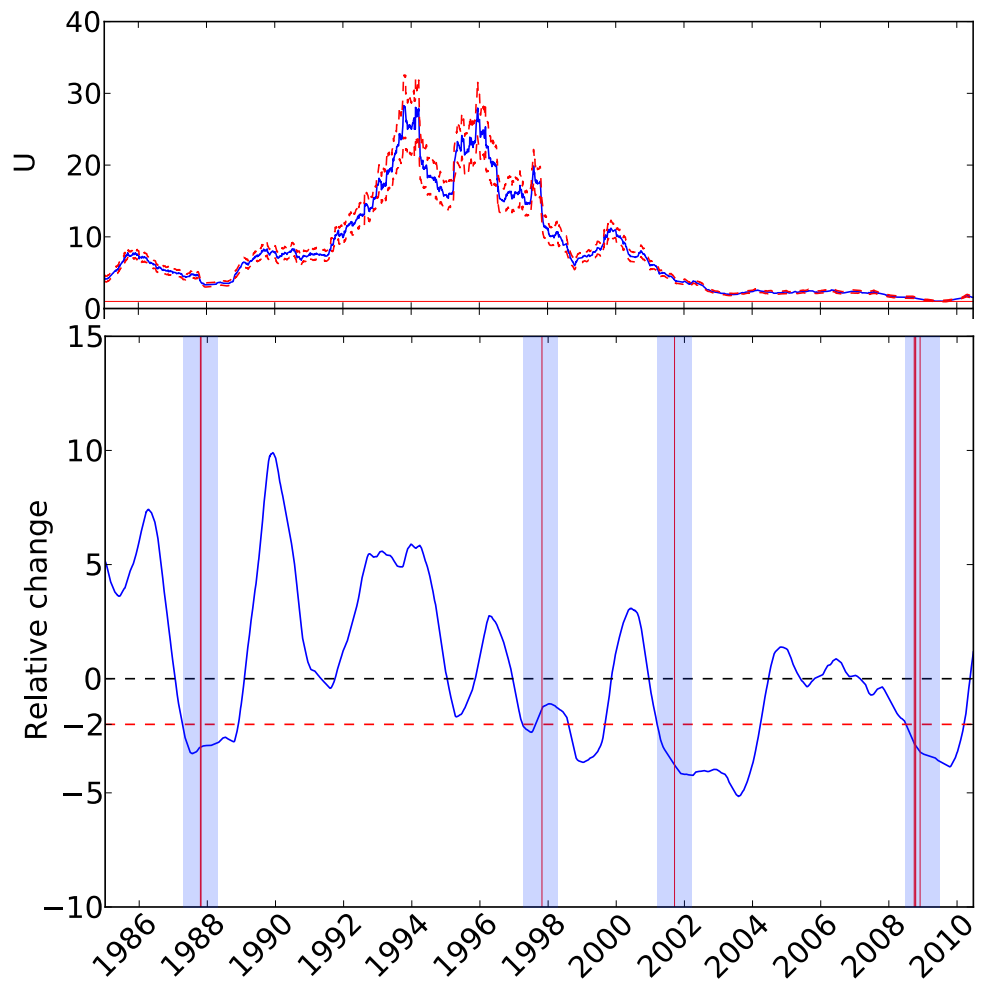

FIG. 3: Top panel is the same as in Figure 2 for the period 1985-2010. Bottom panel is the annual change of $U$ as a fraction of its standard deviation from one year earlier averaged over the previous year. Of the twenty largest percentage drops of the Dow Jones Industrial Average, eight are in the displayed time period: 10/19/1987, 10/26/1987, 10/27/1997, 9/17/2001, 9/29/2008, 10/9/2008, 10/15/2008, 12/1/2008 (vertical red lines). Four year-long windows (shading) follow two standard deviation drops in the the model parameter after periods of increase. Positive-return distributions are computed from the daily returns of stocks of the Russell 3000 for dates after July 1, 1999. Before July 1, 1999, returns were obtained for the Russell 3000 membership lists from 2001, 2004, and 2007. Each positive return fraction was computed with more than 140 stocks.

four non-overlapping, year-long windows).

The performance of the predictive pattern is exceptional. Two questions might be asked to evaluate its robustness. First, the pattern is nearly matched in 1995 when the change of the parameter as a fraction of the standard deviation drops to below -1.67 in April, 1995, but this near match is not followed by a large drop in the DJI within the year. Secondly, the drop in the DJI on September 17, 2001, on the trading day immediately following September 11, 2001, appears to have a direct external cause, and therefore we might not consider the intrinsic stability of the market as predictive, though we do not exclude the possibility that a drop would have occurred without the attack. If we interpret the results conservatively, we would eliminate the year 2001 from consideration $(p<0.0005)$, include the near-prediction 
in $1995(p<0.0004)$, or both $(p<0.002)$. Even in this case, there is strong predictive success.

Our prediction of the event on September 17, 2001 was also obtained by Hurst time series analysis [48], and our work provides additional evidence that this event was not solely a reaction to the events of September 11, but largely reflected intrinsic market dynamics. On the other hand we do not predict an event for 2003. This is to be contrasted with the predictions by others that did not come true [49]. However, we do find a significant drop in $U$ prior to that time, suggesting increased vulnerability. It appears that two events conspire to prevent the crash. First, the increase in mimicry leveled off before the systemic instability threshold. Moreover, following the smaller crash on September 17, 2001 there was no actual recovery of the market dynamics, which continued to be vulnerable, but without a crash, until 2007. Our result that increased mimicry anticipates panics is also distinct from debates about the origins of higher correlations that follow crises [50 52].

Central to the discussion of panic in the literature [1-4] is the degree to which it reflects external threats that cause each individual to panic, or whether it reflects mimicry with or without external causes. Even when mimicry is important, underlying conditions that imply increased risk can elevate sensitivity and the tendency to mimicry. Underlying conditions in this context may include internal trends such as market bubbles or external factors such as war, or the financial disruptions that preceded the recent market decline. When panic involves collective action, rather than individual response, precursor fluctuations are likely to exist due to a growing sensitivity to real or random disturbances. Our results suggest that self-induced panic is a critical component of both the current financial crisis and large single day drops over recent years. The signature we found, the existence of a large probability of co-movement of stocks on any given day, is a measure of systemic risk and vulnerability to self-induced panic. Finally, we note that the ability to distinguish between self-induced panic and the result of external effects may be widely applicable to collective behaviors [53].

Finally, we note that since volatility is often considered a measure of risk, its magnitude might be considered a more natural predictor of crises than the measure of collective following we have presented. Although volatility does increase at the onset of a crisis, and may remain elevated, it provides an unreliable crisis predictor of market crashes. Detailed comparison between our method and volatility will be discussed in a forthcoming paper. [54]

Acknowledgements: We thank James H. Stock, Jeffrey C. Fuhrer and Richard Cooper for 
helpful comments. I.R.E. thanks the Radcliffe Institute for a fellowship. M.A.M.A. thanks FAPESP and CNPq for financial support.

\section{Appendix A: Dynamic network model of daily stock returns}

\section{Model}

Consider a network representing an economic market with $N$ variable nodes taking only the values -1 or 1 , representing decreasing or increasing returns of a particular stock. In addition there are $D$ and $U$ nodes frozen in state -1 and 1 respectively. At each time step a variable node is selected at random; with probability $1-p$ the node copies the state of one of its connected neighbors, and with probability $p$ the state remains unchanged. The frozen nodes are interpreted as external perturbations with negative and positive effects on the returns. Analytically extending $D$ and $U$ to be real numbers enables modeling arbitrary strengths of external perturbations. 42

For a fully connected network the behavior of the system can be solved exactly as follows. The nodes are indistinguishable and the state of the network is fully specified by the number of nodes with internal state 1 . Therefore, there are only $N+1$ distinguishable global states, which we denote $\sigma_{k}, k=0,1, \ldots, N$. The state $\sigma_{k}$ has $k$ variable nodes in state 1 and $N-k$ variable nodes in state -1 . If $P_{t}(m)$ is the probability of finding the network in the state $\sigma_{m}$ at the time $t$, then $P_{t+1}(m)$ can depend only on $P_{t}(m), P_{t}(m+1)$ and $P_{t}(m-1)$. The probabilities $P_{t}(m)$ define a vector of $N+1$ components $\mathbf{P}_{t}$. In terms of $\mathbf{P}_{t}$ the dynamics is described by the equation

$$
\mathbf{P}_{t+1}=\mathbf{T P}_{t} \equiv\left(\mathbf{1}-\frac{(1-p)}{N(N+D+U-1)} \mathbf{A}\right) \mathbf{P}_{t}
$$

where the time evolution matrix $\mathbf{T}$, and also the auxiliary matrix $\mathbf{A}$, is tri-diagonal. The non-zero elements of $\mathbf{A}$ are independent of $p$ and are given by

$$
\begin{aligned}
& A_{m, m}=2 m(N-m)+U(N-m)+D m \\
& A_{m, m+1}=-(m+1)(N+D-m-1) \\
& A_{m, m-1}=-(N-m+1)(U+m-1) .
\end{aligned}
$$


The transition probability from state $\sigma_{M}$ to $\sigma_{L}$ after a time $t$ can be written as

$$
P(L, t ; M, 0)=\sum_{r=0}^{N} b_{r M} a_{r L} \lambda_{r}^{t} .
$$

where $a_{r L}$ and $b_{r M}$ are the components of the right and left $r$-th eigenvectors of the evolution matrix, $\mathbf{a}_{r}$ and $\mathbf{b}_{r}$. Thus, the dynamical problem has been reduced to finding the right and left eigenvectors and the eigenvalues of $\mathbf{T}$.

The eigenvalues $\lambda_{r}$ of $\mathbf{T}$ are given by

$$
\lambda_{r}=1-\frac{(1-p)}{N(N+D+U-1)} r(r-1+D+U)
$$

and satisfy $0 \leq p \leq \lambda_{r} \leq 1$. The equation for $P(L, t ; M, 0)$ shows that the asymptotic state of the network is determined only by the right and left eigenvectors with unit eigenvalue, i.e., by the eigenvectors of $\lambda_{0}=1$. The coefficients of the corresponding (unnormalized) left eigenvector are simply $b_{0 k}=1$. The coefficients $a_{0 k}$ of the right eigenvector are given by the Taylor expansion of the hypergeometric function $F(-N, U, 1-N-D, x) \equiv \sum_{k} a_{0 k} x^{k}$. After normalization these coefficients give the stationary distribution

$$
\rho(k)=\frac{\left(\begin{array}{c}
U+k-1 \\
k
\end{array}\right)\left(\begin{array}{c}
N+D-k-1 \\
N-k
\end{array}\right)}{\left(\begin{array}{c}
N+D+U-1 \\
N
\end{array}\right)} .
$$

This is the probability of finding the network with $k$ nodes 1 in equilibrium and it is independent of the initial state. The other eigenvectors can also be calculated and are also related to hypergeometric functions.

One important feature of this solution is that for $U=D=1$ we obtain $\rho(m)=1 /(N+1)$ for all values of $\mathrm{N}$, i.e., $\mathrm{U}=\mathrm{D}=1$ is the critical value of this model. Thus all states $\sigma_{k}$ are equally likely and the system executes a random walk through the state space. For $U, D>1 \rho(k)$ resembles a Gaussian distribution, but its shape is very different for $U, D<<1$, as illustrated in Figure 4 for $N=500$.

It might seem that the critical point should depend on the size of the external influence relative to the number of nodes in the system, i.e. $U / N$. However, this is an order to disorder transition, and, as with the temperature in physics models of phase transitions, the critical value does not depend on the system size. For all values of $U=D$, the nodes have equal 


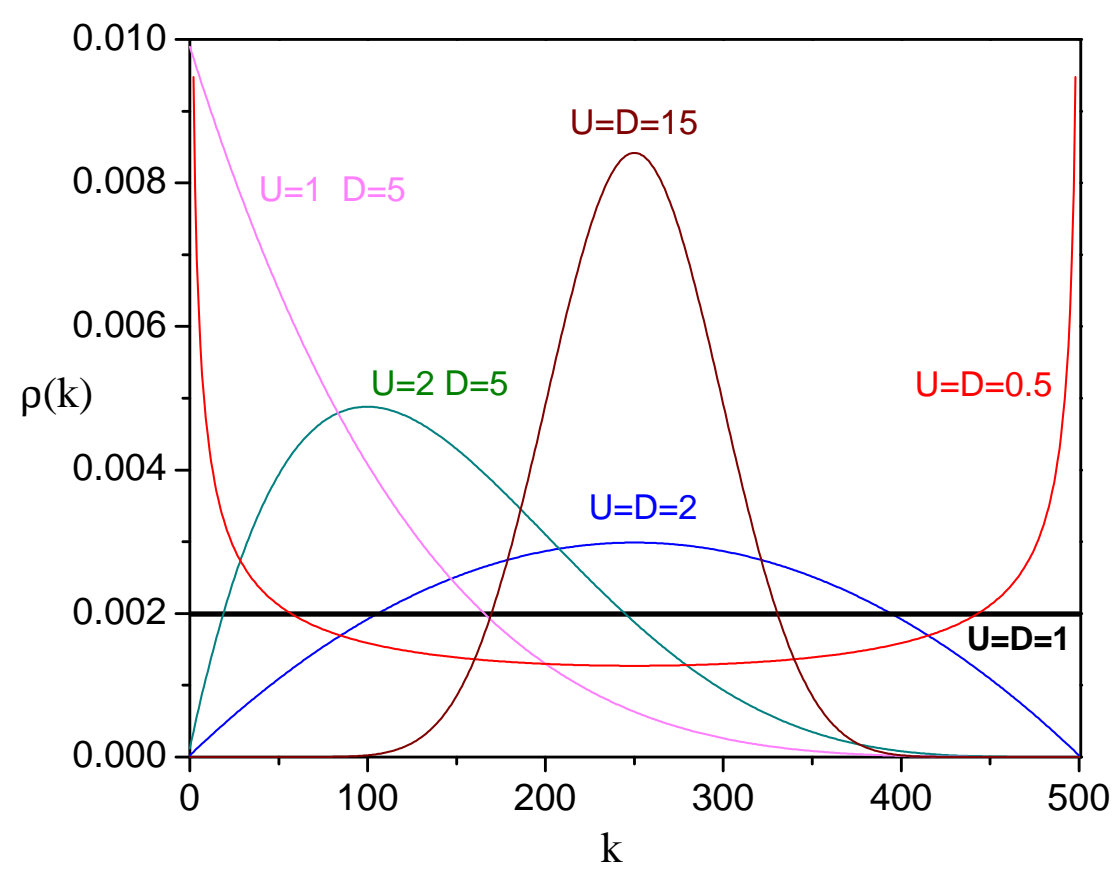

FIG. 4: Probability distributions of finding the network with $k$ nodes 1 in equilibrium for different values of $U$ and $D$. The number of variable nodes is $N=500$

probability of being + or - . Thus, each node experiences an environment that drives it equally toward positive and negative values. The role of the external influence is only as a perturbation promoting the transitions between states of the distribution. In this context, even though the external influence on any one node decreases as $N$ increases, the influence across all nodes is independent of $N$. This is because each node picks the external node to copy in proportion to $1 / N$. Thus, the average number of nodes that are changed per time step by the external influence is independent of $N$.

This model has an analogue in population genetics and can be mapped exactly into the Wright-Fisher-Moran model with two alleles and mutation. 44 Consider a population of $N$ haploid individuals and a gene with alleles $A_{1}$ and $A_{2}$. Sexual reproduction occurs between random pairs of individuals with the offspring replacing one of the expiring parents. After the allele of the offspring is chosen with equal probability between the parents, there is also a probability $\mu_{1}$ to mutate from $A_{1}$ to $A_{2}$ or $\mu_{2}$ to mutate from $A_{2}$ to $A_{1}$. The number of 
alleles $A_{1}$ in the population in equilibrium is given by Equation A1 with

$$
U=\frac{2 \mu_{2}(N-1)}{1-\mu_{1}-\mu_{2}} \quad D=\frac{2 \mu_{1}(N-1)}{1-\mu_{1}-\mu 2}
$$

This problem was first considered by Watterson and Gladstein [55, 56] with no mutation and latter generalized by Cannings [57]. A detailed account is given by Ewens [44].

For networks with different topologies the effect of the frozen nodes is amplified. To see this we note that the probability that a variable node copies a frozen node is $P_{i}=$ $(D+U) /\left(D+U+k_{i}\right)$ where $k_{i}$ is the degree of the node. For fully connected networks

$k_{i}=N-1$ and $P_{i} \equiv P_{F C}$. For general networks an average value $P_{a v}$ can be calculated by replacing $k_{i}$ by the average degree $k_{a v}$. We can then define effective numbers of frozen nodes, $D_{e f}$ and $U_{e f}$, as being the values of $D$ and $U$ in $P_{F C}$ for which $P_{a v} \equiv P_{F C}$. This leads to

$$
D_{e f}=f D, \quad U_{e f}=f U
$$

where $f=(N-1) / k_{a v}$. Therefore, as the network acquires more internal connections and $k_{a v}$ increases, the effective values of $D$ and $U$ decrease.

\section{Curve fits}

Theoretical fits are computed from an unbiased estimator of the standard deviation. The distribution described in Section A 1 takes values $k=0, \ldots, N$. We are interested in the positive fraction, or $k / N$, rather than the number of positive nodes. The central moments of the positive fraction distribution can be computed from Equation A1. We express the mean, $c_{1}$, and variance, $c_{2}$ in terms of $\xi=U /(U+D)$ and $a=U+D$.

$$
\begin{aligned}
& c_{1}=\xi \\
& c_{2}=\frac{\xi(1-\xi)(1+a / N)}{a+1}
\end{aligned}
$$

Equations A3 and A4 can be inverted to solve for $\xi$ and $a$ :

$$
\xi=c_{1}
$$




$$
a=\frac{c_{1}\left(1-c_{1}\right)-c_{2}}{c_{2}-c_{1}\left(1-c_{1}\right) / N}
$$

For the case of stocks, fits using $c_{1}=0.5$ are better fits as measured by the $\chi^{2}$ goodness-of-fit test than fits achieved by setting $c_{1}$ to the mean of the empirical distribution.

\section{Data sources}

To compute empirical distributions, we used daily returns from the Russell 3000, restricted to stocks trading on the NYSE, NYSE Alternext, Nasdaq Capital, and Nasdaq Stock markets. The Russell 3000 is maintained by Russell Investments, and is reconstrustructed every twelve months, with the new composition announced near the end of June. The list includes the largest 3000 US stocks trading on market exchanges by market capitalization. Specific details of the selection process may be obtained from Russell Investments [58].

To compute the empirical distribution of the positive-return fraction, we used two methods. For the period from July 1999 to June 2010, we retrieved daily returns of stocks from the Russell 3000 membership lists published at the end of June for the years 1999 through 2009. Daily returns of the stocks on the list were retrieved for the following twelve month period beginning in July. Stocks that were delisted during this period were included for all days before delisting. For the period before July 1999, we combined ticker symbols from the Russell 3000 membership lists from June 2001, 2004, and 2007, and retrieved daily returns for the symbols back to 1985. Each positive return fraction was computed with more than 140 stocks. 
[1] M. Wolfenstein, Disaster. (Free Press, Glencoe, Ill., 1957).

[2] N. J. Smelser, Theory of Collective Behavior. (Free Press, Glencoe, Ill., 1963).

[3] E. L. Quarantelli, The Sociology of Panic, in International Encyclopedia of the Social and Behavioral Sciences, N. J. Smelser and P. B. Baltes, Eds., (Elsevier, 2001).

[4] A. R. Mawson, Psychiatry 68, 95 (2005).

[5] D.W. Diamond, P.H. Dybvig, Fed. Res. Bank. Minn. Quart. Rev. 24, 14 (2000).

[6] C. W. Calomiris, G. Gorton, in Financial Markets and Financial Crises R. G. Hubbard, Ed. (National Bureau of Economic Research, 1990), chap. 4.

[7] L. Mann, T. Nagel, P. Dowling, Sociometry 39, 223 (1976).

[8] J. K. Galbraith, The Great Crash 1929 (Houghton Mifflin, New York, 1954).

[9] C. Kindleberger, Manias, panics, and crashes (John Wiley \& Sons Inc., New York, 1978).

[10] D. Sornette, A. Johansen, J.-P. Bouchaud, Journal of Physics I (France) 6, 167 (1996).

[11] J. A. Feigenbaum, P.G.O. Freund, Int. J. Mod. Phys. B 10, 3737 (1996).

[12] D. Sornette, A. Johansen, Physica A 245, 411 (1997).

[13] D. Sornette, D. Stauffer and H. Takayasu, in The Science of Disasters: Climate Disruptions, Heart Attacks, and Market Crashes, A. Bunde, J. Kropp and H.J. Schellnhuber, Eds. (Springer, 2002).

[14] D. Sornette, in Extreme Events in Nature and Society, S. Albeverio, V. Jentsch, H. Kantz, Eds. (Springer, 2006).

[15] J. A. Feigenbaum, Quant. Finance 1, 346 (2001).

[16] D. Sornette, A. Johansen, Quant. Finance 1, 452 (2001).

[17] D. S. Bree, N. L. Joseph, arXiv:1002.1010v1 (2010).

[18] A. Cho, Science 325, 408 (2009).

[19] D. Cutler, J. Poterba, and L. Summers, What Moves Stock Prices? Journal of Portfolio Managment, 15, 4 (1989).

[20] G. Morris, The Complete Guide to Market Breadth Indicators: How to Analyze and Evaluate Market Direction and Strength (McGraw-Hill, ISBN-10: 0071444432, 2005).

[21] M. Levy, H. Levy, and S. Solomon, Microscopic Simulation of Financial Markets: From Investor Behavior to Market Phenomena (Academic Press, 2000). 
[22] T. Preis, and H.E. Stanley, J. Stat. Phys. 138431 (2010).

[23] T. Preis, D. Reith, and H. E. Stanley, Phil Trans. R. Soc. A 3685707 (2010).

[24] M. Scheffer, J. Bascompte, W.A. Brock, V. Brovkin, S.R. Carpenter, V. Dakos, H. Held, E.H. van Nes, M. Rietkerk, G. Sugihara, Nature 461, 53 (2009).

[25] S. Ross. The arbitrage theory of capital asset pricing, Journal of Economic Theory 13, 341 (1976).

[26] G. Chamberlain, M. Rothschild, Arbitrage, factor structure, and mean-variance analysis on large asset markets Econometrica /bf 51, 1305 (1983).

[27] R. N. Mantegna, H. E. Stanley, An Introduction to Econophysics. (Cambridge University Press, 2000).

[28] J.-P. Onnela, A. Chakraborti, K. Kaski, J. Kertesz, A. Kanto, Phys. Rev. E 68, 056110 (2003).

[29] R.D. Smith, J. Korean Phys. Soc. 54, 2460 (2009).

[30] P. Jorion, Value at Risk: The New Benchmark for Managing Financial Risk, 3rd Ed. (McGrawHill, 2006).

[31] D. Harmon, B. Stacey, Yavni Bar-Yam, and Yaneer Bar-Yam, Networks of Economic Market Interdependence and Systemic Risk. arXiv:1011.3707v2 (2010)

[32] F. Black, Studies of stock price volatility changes, Proceedings of the 1976 Meetings of the American Statistical Association, Business and Economical Statistics Section, 177 (1976)

[33] A. A. Christie, The stochastic behavior of common stock variances: value, leverage and interest rate effects, Journal of Financial Economics 10, 407 (1982)

[34] D. Nelson, Conditional Heteroskedasticity in Asset Returns: A New Approach, Econometrics 45, 347 (1991).

[35] G. Bekaert, G. Wu, Asymmetric volatility and risk in financial markets, Review of Financial Studies 13, 1 (2000)

[36] G. Wu, The Determinants of Asymmetric Volatility (Social Science Resource Network, 2001; http://ssrn.com/abstract=248285).

[37] J. B. De Long, A. Shleifer, L. H. Summers, R. J. Waldmann, J. Finance 45, 379 (1990).

[38] J. B. De Long, A. Shleifer, L. H. Summers, R. J. Waldmann, J. Polit. Econ. 98, 703 (1990).

[39] D.S. Scharfstein, and J.C. Stein, The Am. Econ. Rev. 80, 466 (1990).

[40] S. Bikhchandani, D. Hirshleifer, and I. Welch, J. Econ. Persp. 12, 151 (1998).

[41] F. Schweitzer, G. Fagiolo, D. Sornette, F. Vega-Redondo, A. Vespignani, D.R. White, Science 
325, 422 (2009).

[42] D. D. Chinellato, M. A. M. de Aguiar, I. R. Epstein, D. Braha, Y. Bar Yam, arXiv:0705.4607v2 (2007).

[43] W.B. Arthur, in The economy as an evolving complex system. Eds. P.W. Anderson, K.J. Arrow, D. Pines, (Addison-Wesley, 1988).

[44] W.J. Ewens, Mathematical Population Genetics I. Theoretical Introduction Series: Biomathematics, Vol. 9 (New York: Springer Verlag, 1979).

[45] Dow Jones Averages. Dow Jones Industrial Average, Milestones. http://www.djaverages. com/?view=industrial\&page=milestones (accessed July 8, 2010).

[46] M. Carlson, A Brief History of the 1987 Stock Market Crash with a Discussion of the Federal Reserve Response. (Finance and Economic Discussion Series, Divisions of Research \& Statistics and Monetary Affairs Federal Reserve Board, Washington, D. C., (2007).

[47] A. Berg, International Monetary Fund Working Paper WP/99/138 (1999).

[48] D. Grech, Z. Mazur, Physica A 336, 133 (2004).

[49] D. Sornette, Why Stock Markets Crash. (Princeton University Press, 2003).

[50] F. Longin and B. Solnik, Journal of Finance 56, 649, (2001).

[51] K. J. Forbes, R. Rigobon, Journal of Finance 57, 2223 (2002).

[52] G. M. Caporale, A. Cipollini and N. Spagnolo, Journal of Empirical Finance 12, 476 (2005).

[53] Y. Bar Yam, Dynamics of Complex Systems (Perseus Press, Reading, 1997).

[54] M Lagi, M. A. M. de Aguiar, and Y. Bar-Yam, Comparison of volatility and following as predictors of market crises (in preparation).

[55] Watterson, G.A. Markov Chains with Absorbing States: A genetic example. The Annals of Mathematical Statistics 32, 716 (1961).

[56] Gladstein, K. The Characteristic Values and Vectors for a Class of Stochastic Matrices Arising in Genetics. Siam J. Appl. Math 34, 630 (1978).

[57] Cannings, C. The latent roots of certain Markov chains arising in genetics; a new approach. 1. Haploid models. Adv. Appl. Prob. 6, 260 (1974).

[58] Russell Investments, Russell U.S. Equity Indexes Construction and Methodology. http:// WwW.russell.com/indexes/documents/Methodology.pdf (accessed July 13, 2010). 